\title{
Analysis of RecA-independent recombination events between short direct repeats related to a genomic island and to a plasmid in Escherichia coli K12
}

\author{
María F Azpiroz $^{\text {Corresp., }}{ }^{1}$, Magela Laviña ${ }^{1}$ \\ ${ }^{1}$ Fisiología y Genética Bacterianas, Facultad de Ciencias, Universidad de la República, Montevideo, Uruguay \\ Corresponding Author: María F Azpiroz \\ Email address: fernanda@fcien.edu.uy
}

RecA-independent recombination events between short direct repeats, leading to deletion of the intervening sequences, were found to occur in two genetic models in the Escherichia coli K12 background. The first model was a small $E$. coli genomic island which had been shown to be mobile in its strain of origin and, when cloned, in the E. coli $\mathrm{K} 12$ context too. However, it did not encode a site-specific recombinase as mobile genomic island usually do. Then, it was deduced that the host cells should provide the recombination function. This latter was searched for by means of a PCR approach to detect the island excision in $E$. coli K12 mutants affected in a number of recombination functions, including the $16 \mathrm{E}$. coli $\mathrm{K} 12$ site-specific recombinases, the RecET system, and multiple proteins that participate in the RecA-dependent pathways of homologous recombination. None of these appeared to be involved in the island excision. The second model, analyzed in a RecA deficient context, was a plasmid construction containing a short direct repeat proceeding from Saccharomyces cerevisiae, which flanked the cat gene. The excision of this gene by recombination of the DNA repeats was confirmed by PCR and through the detection, recovery and characterization of the plasmid deleted form. In sum, we present new evidence on the occurrence of RecA-independent recombination events in E. coli K12. Although the mechanism underlying these processes is still unknown, their existence suggests that RecA-independent recombination may confer mobility to other genetic elements, thus contributing to genome plasticity. 
1

2

3

Analysis of RecA-independent recombination events

4

between short direct repeats related to a genomic island and to a plasmid

5

in Escherichia coli $\mathrm{K} 12$

6

7

8

María F. Azpiroz* and Magela Laviña

9

10

Fisiología y Genética Bacterianas, Facultad de Ciencias, Universidad de la República,

Montevideo, Uruguay

12

13

$14 *$ Corresponding author:

15 María Fernanda Azpiroz

16 E-mail: fernanda@fcien.edu.uy

17 Tel. 59825258618 ext. 233

$18 \quad$ Fax. 59825258629

19

20 Abstract 
21 RecA-independent recombination events between short direct repeats, leading to deletion of the

22 intervening sequences, were found to occur in two genetic models in the Escherichia coli K12

23 background. The first model was a small E. coli genomic island which had been shown to be

24 mobile in its strain of origin and, when cloned, in the E. coli K12 context too. However, it did

25 not encode a site-specific recombinase as mobile genomic island usually do. Then, it was

26 deduced that the host cells should provide the recombination function. This latter was searched

27 for by means of a PCR approach to detect the island excision in E. coli K12 mutants affected in a

28 number of recombination functions, including the 16 E. coli $\mathrm{K} 12$ site-specific recombinases, the

29 RecET system, and multiple proteins that participate in the RecA-dependent pathways of homologous recombination. None of these appeared to be involved in the island excision. The second model, analyzed in a RecA deficient context, was a plasmid construction containing a short direct repeat proceeding from Saccharomyces cerevisiae, which flanked the cat gene. The excision of this gene by recombination of the DNA repeats was confirmed by PCR and through the detection, recovery and characterization of the plasmid deleted form. In sum, we present new evidence on the occurrence of RecA-independent recombination events in E. coli $\mathrm{K} 12$. Although the mechanism underlying these processes is still unknown, their existence suggests that RecA-independent recombination may confer mobility to other genetic elements, thus contributing to genome plasticity.

Introduction 
44 Bacterial genomes contain short repeated DNA sequences, which are prone to recombine and

45

46

47

48

49

generate genetic rearrangements. Some of them are associated with mobile genetic elements such as prophages and genomic islands, whose insertion generates short, direct repeats on either side of the element. These flanking repeats, known as attachment sites, are usually the target of dedicated site-specific recombinases that excise these elements so that they remain as independent covalently closed circular molecules. In the inverse reaction, the same recombinases integrate the circular phage or island into the corresponding attachment site in the genome (Hallet \& Sherratt, 1997; Hochhut et al., 2006; Lin et al., 2008; Murphy \& Boyd, 2008; Juhas et al., 2009).

The present work continues previous studies on the mobility of a genomic island, called H47 (H47 GI), contained in the chromosome of an Escherichia coli strain (H47). It is a small $13 \mathrm{~kb}-$ genetic element devoted to the production of the antibacterial peptide microcin H47 (Laviña, Gaggero \& Moreno, 1990; Poey, Azpiroz \& Laviña, 2006). The H47 GI was found to be an unstable mobile element, able to excise from the chromosome by recombination between its attachment sites ( $a t t L$ and $a t t R$ ) in a process generating two products: the chromosome without the island and the island as an independent covalently closed circular molecule (Figure 1A) (Azpiroz, Bascuas \& Laviña, 2011). A DNA segment containing the island had been cloned into several multi-copy plasmid vectors and introduced into E. coli K12 cells. In this foreign background, the H47 GI retained its mobility as in the original H47 strain. In addition, it was found that the excised island was able to integrate into another plasmid provided that it contained the corresponding attachment site. These findings were attained by means of PCR experiments, which allowed the detection of both recombinant sequences generated from each type of recombination event, excision and integration (Azpiroz, Bascuas \& Laviña, 2011). The H47 GI 
67 is flanked by two extensive and imperfect direct repeats, attL and attR, which share four main

68 regions of homology (Figure 1B). In the case of excision, attL and attR recombine and the two

69 resulting products were detected by the appearance of amplicons that included the recombined

70 attachment sites: attC in the deleted replicon and attI in the excised H47 GI (Figure 1C).

71 Amplicons containing the $a t t C$ site were a mix of four sequences corresponding to recombination

72 at the four main regions of homology between attL and attR. This recombination pattern

73 remained unchanged when the analysis was carried out in RecA-deficient E. coli K12 cells,

74 indicating that the H47 GI mobility is a process independent from the homologous

75 recombination pathway (Figures 1C and S1) (Azpiroz, Bascuas \& Laviña, 2011).

76 Many reports refer that genomic islands usually undergo mobility events, and that these are

77 catalyzed by site-specific recombinases encoded by the islands themselves, a feature that is

78 shared with temperate phages (Hochhut et al., 2006; Juhas et al., 2009). However, the H47 GI

79 does not encode any recombinase: when most of its DNA was deleted, recombination between

80 its attachment sites remained unchanged (Azpiroz, Bascuas \& Laviña, 2011). Therefore, the

81 genomic background of $E$. coli $\mathrm{H} 47$ and of $E$. coli $\mathrm{K} 12$ should provide the recombination

82 function responsible for the H47 GI mobility. The island would thus employ a sort of parasitic

83 strategy and, given the characteristics of the rearrangements observed, a site-specific

84 recombination process was expected to accomplish the genetic exchange.

85 In this work, we searched for the function underlying the H47 GI mobility, working with the

86 cloned island in E. coli K12 cells. The H47 GI excision was PCR-assayed in a broad collection

87 of mutants affected in the site-specific and homologous recombination pathways encoded by $E$.

88 coli $\mathrm{K} 12$. None of these functions affected the island mobility, indicating that the process under

89 study could be included into the poorly-known group of RecA-independent recombination events 
90 (Bzymek \& Lovett, 2001). However, we could not succeed to obtain a clone carrying the deleted

91 form with the attC site. To gain further insight into this type of process, a plasmid construction

92 containing the repeated FRT (Flp recognition target) site from Saccharomyces cerevisiae was

93 similarly analyzed in the context of E. coli K12. In this model, RecA-independent deletion of the

94 intervening sequence between the repeats was detected in vitro by PCR and in vivo by the

95 appearance of the deleted form in sufficient amounts to be recovered from plasmid DNA

96 preparations.

Materials \& Methods

99

100

101

102

103

104

105

106

107

108

109

110

111

\section{Bacterial strains, plasmids, and growth conditions}

E. coli $\mathrm{K} 12 \mathrm{BZB} 1011$ is a spontaneous nalidixic acid resistant derivative of W3110, a strain whose genome has been sequenced (GenBank: AP_009048). Most strains used are BZB1011 derivatives, carrying deletions proceeding from selected mutants of the Keio Collection (Baba et al., 2006). In these latter, gene deletions with replacement by a kanamycin resistance (kan) cassette were introduced into BZB1011 by P1 transduction, selecting the kanamycin resistant clones (Table S1). When required, double deletion mutants were constructed in two steps: the kanamycin cassette of the first mutated gene was eliminated as indicated previously (Datsenko \& Wanner, 2000) and the cured mutant was then transduced with a P1 grown on the second deletion mutant, selecting the kanamycin resistant clones. Multiple deletion mutants were constructed with further rounds of the same procedure. E. coli $\mathrm{K} 12 \mathrm{JC} 8679$ is a $r e c B$ rec $C$ sbc $A$ mutant (Coli Genetic Stock Center strain 6490). Two mutant derivatives of BW25113, the parent E. coli K12 strain for the Keio Collection, were used: BW25113 recA56 and BW25113 topB 
$112 \operatorname{rec} A 56$, this latter bearing the $\triangle t o p B 761:: k a n$ mutation from the Keio Collection. The recA56

113 allele was introduced by transducing BW25113 and BW25113 topB (JW1752) with P1 grown on

114 a srl::Tn10 recA56 strain from our laboratory collection, selecting the tetracycline resistant

115 clones and then assaying the recA defficiency by testing their UV sensitivity.

116 Plasmid pEX2000 is a pBR322 derivative that carries the H47 GI included in a 16,823 bp-

117 chromosomal DNA segment from strain H47 (GenBank: AJ_009631). Plasmid p_int1 carries the 118 same DNA segment but deleted for most of the H47 GI, keeping the attachment sites and the 119 external chromosomal adjacent sequences (Azpiroz, Bascuas \& Laviña, 2011). pUY-FRT was

120 constructed by cloning a 1,182 bp HindIII fragment from pKD3 (GenBank: AY_048742) into the

121 pUC13 vector. The insert contains the chloramphenicol resistance gene (cat) flanked by the FRT

122 sites from $S$. cereviseae. Since pKD3 requires the pir gene product to be provided in trans for its 123 replication (Datsenko \& Wanner, 2000), pUY-FRT was constructed to avoid this requirement.

124 Bacteria were grown in LB medium at $37^{\circ} \mathrm{C}$. Antibiotics were added at the following final 125 concentrations: kanamycin, $30 \mu \mathrm{g} / \mathrm{ml}$; ampicillin, $50 \mu \mathrm{g} / \mathrm{ml}$, chloramphenicol, $60 \mu \mathrm{g} / \mathrm{ml}$, and 126 tetracycline, $20 \mu \mathrm{g} / \mathrm{ml}$.

127 Plasmid purification, PCR assays and DNA sequencing

128 Plasmid DNA was extracted with “QIAprep Spin Miniprep Kit” (Qiagen). DNA bands were 129 extracted from gels using "QIAEXII Gel extraction kit” (Qiagen). For PCR reactions, primers 130 and conditions employed are explained in Table 1. For sequencing purposes, PCR products were 131 extracted from gels as explained. Both strands of the OUT and IN amplicons from the pUY-FRT 132 model were sequenced using the primers employed for their amplification. DNA sequencing was 133 performed at the "Molecular Biology Unit" of the Pasteur Institute of Montevideo. 


\section{Results}

136

137

138

139

140

141

142

143

144

145

146

147

148

149

150

151

152

153

154 155

\section{Excision of the $\mathrm{H} 47$ genomic island}

Recombination underlying the H47 GI mobility was studied through the detection of the island excision from a multi-copy recombinant plasmid, pEX2000, in the context of E. coli K12 cells. Plasmid pEX2000 carries a DNA segment from the E. coli H47 chromosome containing the H47 GI flanked by its direct repeats and by adjacent chromosomal sequences on both sides. The direct repeats, attL (148 bp) and attR (144 bp), are imperfect and share four main segments of homology of 13, 11, 20 and $17 \mathrm{bp}$ (Figure 1B). Recombination between them leads to the excision of the H47 GI, which is assessed by PCR using primers "out" in order to detect the occurrence of the empty attC site (amplicon $\mathrm{OUT}_{\mathrm{H} 47}$ ) (Figure 1A). The assays were performed in the context of the E. coli K12 strain BZB1011 and in a set of BZB1011 derivative mutants affected in recombination functions (Table S1). The purpose was to identify the mutant/s where excision did not occur, thus revealing the gene/s involved in the recombination process. Plasmid pEX2000 was introduced into each of these strains and then was extracted to be used as template in PCR reactions. The sequence of the OUT amplicons corresponding to the mutant strains was compared with that from the wild-type BZB1011 strain, i.e. with a mix of four overlapping sequences with the predominance of that corresponding to recombination in site III (Figure S1).

Considering that genomic islands usually employ site-specific recombinases to mediate their mobility, the search first concentrated in mutant strains deficient for such enzymes. Although the H47 GI attachment sites did not reveal significant homology with any known target for a sitespecific recombinase, we did not discard this possibility since it has been reported that some of 
156 these enzymes are able to work -although with low efficiency- on secondary sites that differ in

157 their sequence from the primary one (Menard \& Grossman, 2013). Therefore, the excision of the

158 H47 GI was analyzed in mutants deficient for each of the 16 site-specific recombinases encoded

159 by the $E$. coli $\mathrm{K} 12$ chromosome, most of them related to prophages (int $F$, intD, $y b c K$, intE, pin,

160 intR, pinR, pinQ, intQ, int $S$, int $A$, xerD, xerC, intB, fimB and fimE). Mainly based on homologies

161 among these enzymes, double and multiple mutants were constructed and included in the assays

162 (Table S1). In all cases the $\mathrm{OUT}_{\mathrm{H} 47}$ amplicon appeared and always revealed the same pattern of

163 overlapping sequences as that proceeding from the wild-type BZB1011 strain (Figure S1).

164 Given the previous results, the involvement of site-specific recombination in the H47 GI

165 mobility was discarded. An important fact was that recombination always took place in regions

166 of total homology between the attachment sites, indicating that this condition would be

167 necessary. Therefore, we examined the possible involvement of several genes that participate in

168 homologous recombination.

169 First, the RecA-independent RecET homologous recombination pathway, encoded by the

170 defective Rac prophage present in E. coli K12, was analyzed (Kolodner, Hall \& Luisi-DeLuca,

171 1994). It was considered that the recET genes might have a basal level of expression, as has been

172 proposed for E. coli K12 crytic prophage genes (Wang et al., 2010). The excision of the H47 GI

173 was assayed in BZB1011 mutant derivatives recE (deficient for exonuclease VIII) and recT

174 (deficient for the recombinase function). None of these contexts affected the recombination

175 pattern. The same result appeared using the $\operatorname{rec} B$ recC $s b c A$ strain JC8679, a genetic background

176 where the RecET pathway is known to be induced (Kolodner, Hall \& Luisi-DeLuca, 1994). 
177 It was also considered that recombination under study, although RecA-independent, could share

178 some functions with the homologous recombination pathways. Following this idea, the excision

179 of the H47 GI was surveyed in a set of mutants defective for proteins that participate in

180 homologous recombination ( $r e c B, r e c C, r e c D, r e c F, r e c O, r e c R, r e c N, r e c J$, rec $Q$, recG, $s b c B$,

$181 s b c C, s b c D, u v r D, \operatorname{ruv} A, \operatorname{ruv} B, \operatorname{ruv} C, \operatorname{rad} A, \operatorname{rec} X, \operatorname{exo} X, \operatorname{rar} A, \operatorname{seq} A$ and helD). The double mutant

182 recT recA, deficient for all known homologous recombination pathways, was constructed and

183 also included in the assays. The result was that in neither mutant the excision of the island was

184 detectably affected: the $\mathrm{OUT}_{\mathrm{H} 47}$ product always appeared as a weak band in gels and its sequence

185 exhibited the same pattern as that of the amplicon from the wild-type strain (Figure S1).

186 Therefore, none of the functions analyzed related to homologous recombination appeared to be

187 involved in the H47 GI mobility.

\section{In vivo search for the H47 GI excision}

189 Although in vitro PCR experiments consistently supported the existence of recA-independent

190 recombination events between the attL and attR sites flanking the H47 GI, this phenomenon still

191 awaited an in vivo confirmation. This would imply the detection and characterization of at least a

192 clone carrying the replicative recombination product, i.e. the deleted molecule. Obviously, the in

193 vivo detection of the other putative product, the excised circular form, would be much less likely

194 because of the non-replicative condition of this molecule. It should be noted that the model of the

$195 \mathrm{H} 47$ genomic island was rather complex since it encodes the production of an antibiotic activity,

196 which would exert a deleterious effect upon cells that had lost the island in a recombination

197 event. For this reason, we investigated this phenomenon not only in strains carrying the entire

198 island (pEX2000) but also in strains with a plasmid derivative in which most of the intervening

199 sequence between the att sites had been deleted (p $\Delta$ int 1$)$. 
200 After being propagated in $E$. coli $\mathrm{K} 12$ cells (BZB1011 recT recA), plasmids were extracted, and 201 important amounts of DNA (1-2 $\mu \mathrm{g})$ were run in gels in an attempt to perceive new bands that

202 could correspond to the recombination products. We did not succeed to see anything other than 203 the plasmids' original forms.

204 It has been described that E. coli mutants affected in the topB gene, encoding topoisomerase III,

205 have an increased frequency of spontaneous deletions occurring at short direct repeats

206 (Whoriskey, Schofield \& Miller, 1991; Schofield et al., 1992; Uematsu, Eda \& Yamamoto,

207 1997). Therefore, we repeated the assays in the context of strains carrying a top $B$ deficient allele.

208 BZB1011 topB was constructed, but its $r e c A$ derivative resulted nonviable. Considering that the

209 topB context would be affected in DNA supercoiling, the nalidixic acid resistant strain BZB1011

210 might not be suitable for this study since it most probably contains a mutant DNA gyrase. For

211 this reason, the gyr wild type BW25113 context was employed. BW25113 recA and

212 BW25113topB recA were constructed and transformed with pEX2000 and $\mathrm{p} \Delta \mathrm{int} 1$. In all these

213 strains, although recombination between the att H47 sites was PCR-confirmed, no extra-bands

214 appeared when plasmids' DNA was analyzed in gels.

215 H47 GI-related attC sequence survey in data banks

216 Since no product of the H47 GI excision could be detected in vivo, a clue in this sense was

217 searched for in data banks. This type of survey had been done before, mainly focusing on the 218 presence of the H47 GI in other strains besides E. coli H47 (Azpiroz, Bascuas \& Laviña, 2011).

219 Now, we looked for the four types of attC sequences (I-IV), corresponding to recombination

220 events in each of the four segments of identity between the attL and attR sites. The search

221 revealed that these sequences are widespread among pathogenic E. coli strains and also appear in

222 two strains of Salmonella enterica, always being present in the chromosome. Although these 
223 sequences exhibited a certain degree of variability, several attC sites could be recognized and

224 distinguished from $a t t L$ and $a t t R$. They were further confirmed as $a t t C$ by analyzing their

225 adjacent sequences (about 500 nucleotides on each side), which should correspond to those

226 surrounding the $\mathrm{H} 47 \mathrm{GI}$. Most of them were $a t t C_{\mathrm{III}}$, resulting from recombination at the most

227 extensive site of homology between the direct repeats. Among these, six identical matches were

228 found (E. coli strains Sanji, SEC470, RS76, PCN033, Santai O157:H16, and S. enterica

229 Heidelberg str. SL476). There was a match with $\operatorname{att}_{\mathrm{II}}($ E. coli ETEC H10407) and another single

230 match with $a t t C_{\mathrm{IV}}$ (E. coli FHI23). No sequence of the $\operatorname{att} C_{\mathrm{I}}$ type appeared. Therefore, these

231 findings strongly supported the idea that the H47 GI is indeed a mobile element due to

232 recombination between its attachment sites.

233

234

235

236

237

238

239

240

241

242

243

244

\section{RecA-independent recombination in the model of plasmid pUY-FRT}

In view that our efforts to detect in vivo the H47 GI excision were unsuccessful, the studies were extended to a simpler genetic model. It was a multi-copy plasmid, pUY-FRT, carrying the cat gene (for chloramphenicol resistance) flanked by a perfect 46 bp-direct repeat. The repeated sequences proceeded from $S$. cereviseae and contained the FRT target for the site-specific recombinase Flp. Recombination between the repeats would lead to the deletion of the intervening cat-containing DNA segment. The assays were carried out in the absence of the Flp enzyme in E. coli K12 cells. Specifically, plasmid pUY-FRT was propagated and then extracted from BZB1011 recT recA, BW25113 recA and BW25113 topB recA.

The experiments began by transforming with pUY-FRT the three E. coli K12 mutant backgrounds and chloramphenicol resistant transformants were selected so as to ensure that the assays started with clones carrying the original non-deleted form of the plasmid. After isolation 
245 in the same medium, clones were grown in liquid LB Ap, which was supposed to be a permissive

246 condition for the propagation of the deleted plasmid.

247 First, plasmid DNA was used as template in PCR reactions devoted to detect the excision of the

248 DNA segment between the repeats, i.e. the cat gene. As with the H47 GI, two types of reactions

249 were carried out to detect the two possible products: an $\mathrm{OUT}_{\mathrm{FRT}}$ amplicon of $381 \mathrm{bp}$ in the

250 deleted plasmid, and an $\mathrm{IN}_{\mathrm{FRT}}$ amplicon of $335 \mathrm{bp}$ in the excised circular form. In both types of

251 reactions, amplicons of the expected size were produced and appeared in the three contexts

252 assayed. The DNA sequence of these PCR products exhibited the expected recombination

253 pattern, i.e. a single repeat flanked by recombined sequences.

254 For in vivo analysis, ca. 500 ng of plasmid DNA were loaded in gels. This time, a new small

255 band was barely seen in plasmid preparations proceeding from the top $B^{+}$backgrounds, being

256 more evident in the BW25113 recA context, and this same band appeared clearly stronger when

257 the plasmid came from the top $B$ deficient cells. We presumed that it could correspond to the

258 deleted plasmid molecule, which would be called pUY-FRT $\Delta$ (Figure 2A). Then, this smaller

259 band, proceeding from BW25113 recA and BW25113 topB recA, was extracted from gels and

260 used to transform BW25113 recA with selection for ampicillin-resistance. In each case, a few

261 clones grew and, when assayed in the presence of chloramphenicol, all proved to be sensitive.

262 Plasmid DNA was extracted from a clone of each type of transformant and was then digested

263 with EcoRI and HindIII. Both plasmids were found to be identical in their size and restriction

264 profile, which corresponded to those of a deleted plasmid derived from pUY-FRT by

265 recombination between its direct repeats (Figure 2B). DNA sequencing confirmed this structure:

266 the sequence was read through the recombined repeat and beyond it more than $300 \mathrm{bp}$ on each

267 side. 
268 Finally, it should be mentioned that no trace of the excised DNA could be seen.

269

270

271

272

273

274

275

276

277

278

279

280

281

282

283

284

285

286

287

288

289

\section{Discussion}

The analysis of the mobility of an E. coli small genomic island led us to study RecA-independent recombination phenomena. Specifically, the H47 GI presented the peculiarity of being able to excise from its site of insertion although it did not code for a cognate integrase. Moreover, its mobility was not affected in a RecA deficient background (Azpiroz, Bascuas \& Laviña, 2011). It should be mentioned that a few other genetic elements have been described to be mobile and to lack a recombinase gene (Santiviago et al., 2010; Palmiere, Mingoia \& Varaldo, 2013).

The fact that the H47 GI mobility could be analyzed in the E. coli K12 genetic context appeared as an excellent opportunity to identify the host function involved in the genetic exchange. The availability of the genome sequence of $E$. coli $\mathrm{K} 12$ as well as of an exhaustive collection of derivative deletion mutants encouraged this presumption. However, after assaying the H47 GI excision in a number of mutants affected in recombination functions, including the 16 E. coli K12 site-specific recombinases, the RecET system, and multiple proteins that participate in the RecA-dependent pathways of homologous recombination, it was concluded that none of these processes appeared to be involved and that an unknown RecA-independent recombination mechanism would be responsible for the H47 GI mobility. Anyway, our results could harbor some uncertainty since the PCR technique has been described to generate chimeric products when the template contains repeated sequences, an artifact that has been detected in the amplification of 16S rRNA genes in metagenomic studies (Wang \& Wang, 1997). Although this possibility seems rather unlikely, we cannot completely discard it. 
290 Therefore, an in vivo demonstration of the existence of the H47 GI mobility was searched for.

291 This was particularly difficult given the lack of selection for the recombined clones and that the

292 frequency of recombination appeared to be low. In fact, we were unable to obtain a clone

293 carrying a recombination product, i.e. an $a t t C$ site in a replicon that had previously carried the 294 H47 GI.

295 Other authors have analyzed the mobility of some genetic elements that lack a recombinase gene

296 by means of PCR experiments similar to those employed in this work (Palmieri, Mingoia \&

297 Varaldo, 2013). These elements also have in common that their attachment sites are very

298 extensive, of several hundred bp. In two cases, a defective prophage-like element from $S$.

299 enterica serovar Enteritidis and a transposon from Streptococcus pneumonia, clones that

300 spontaneously lost these elements could be isolated. Interestingly, their excision was PCR-

301 detected in a RecA-deficient background (Santiviago et al., 2010; Palmieri et al., 2012). In view

302 of these previous descriptions, we presume that the length of the attachment sites and their level

303 of identity may be an important factor that determines the frequency at which recombination

304 takes place and, thus, the likelihood of its in vivo detection. The two mentioned examples from

305 S. enterica and S. pneumonia have attachment sites of $308 \mathrm{bp}$ (98\% of identity) and ca. $1200 \mathrm{bp}$

306 ( $>99 \%$ of identity), respectively. For their part, the H47 GI repeats have clearly smaller numbers:

$307146 / 8 \mathrm{bp}$ in length with $77 \%$ of identity, the most extensive perfect homology being of $20 \mathrm{bp}$ (see

308 Figure 1B). In other previous descriptions of RecA-independent recombination events, it was

309 also found that homology was required and that recombination rates increased with the length of

310 the repeats (Albertini et al., 1982; Bi \& Liu, 1994). Another factor that could hinder the detection

311 of recombined molecules was that the H47 GI encodes an antibacterial activity, microcin H47,

312 together with it cognate immunity. Those cells harboring deleted molecules by loss of the island 
313 would be in a clear disadvantage compared to other cells because they would be more sensitive

314 to the microcin. Nevertheless, when we worked with a plasmid construction lacking most of the

315 island content, recombinant molecules could not be detected either.

316 Finally, a survey performed in data banks searching for H47 GI-related attC sequences revealed

317 that these empty attachment sites exist in natural strains. Representatives of three of the four

318 possible versions of recombination between the direct repeats were found. These findings show

319 that the H47 GI is indeed a mobile genetic element whose presence in the bacterial chromosome

320 is unstable. Although these empty attachment sites would represent traces of recombination

321 events between the direct repeats, they do not ensure the widespread occurrence of this type of

322 process in the E. coli species: the possibility that recombination could only take place in some

323 genetic backgrounds providing a specific recombinase cannot be discarded.

324 At this point, we wondered how general this recombination phenomenon could be and then

325 decided to study a different genetic model, plasmid pUY-FRT, in the context of E. coli K12. This

326 model is much simpler than the H47 GI and contains perfect and longer direct repeats of $46 \mathrm{bp}$

327 flanking the cat gene. As in the case of the island, excision events were detected by a PCR

328 approach in wild type and in $r e c A$ - and $\operatorname{rec} T$-deficient contexts, and the genetic exchange always

329 occurred at the pUY-FRT repeats. However, unlike what happened with the island, this model

330 allowed the detection of a deleted derivative molecule which had lost the cat gene by

331 recombination between the direct repeats. In fact, this is probably the first time that a RecA-

332 independent recombination product is readily detected in a plasmid preparation from a culture

333 that had been seeded with a strain carrying the original plasmid form. This phenomenon

334 appeared to be enhanced in a topB mutant, deficient for topoisomerase III, a context that has

335 been previously described to increase some RecA-independent recombination events 
336 (Whoriskey, Schofield \& Miller, 1991; Schofield et al., 1992; Uematsu, Eda \& Yamamoto,

337 1997). Therefore, in the model of pUY-FRT we succeeded to demonstrate that a RecA-

338 independent recombination mechanism is able to work on short direct repeats in E. coli K12.

339 These results support the idea that similar events could indeed happen in the H47 GI but, in this

340 case, their frequency would be too low to be detected in vivo. There are several differences

341 between the two models analyzed that could determine differences in their excision rate. For

342 instance, it has been claimed that the longer the extent of perfect homology between the repeats

343 and the shorter the distance separating them, the higher the rate of recombination (Albertini et

344 al., 1982; Bi \& Liu, 1994; Lovett et al., 1993; Lovett et al., 1994). These two factors could

345 explain the higher frequency of excision in pUY-FRT in relation to that in H47 GI. pUY-FRT

346 has $46 \mathrm{bp}$-long perfect direct repeats separated by an intervening sequence of $886 \mathrm{bp}$, while the

347 H47 GI has perfect repeats of 13, 11, 20 and 17 bp, separated by 12,635 bp. Apparently, the

348 condition of the DNA repeats would be predominant, considering that recombination between

349 the H47 GI repeats in plasmid $\mathrm{p} \Delta$ int 1 could not be detected in vivo even though the intervening

350 sequence was shortened to $554 \mathrm{bp}$, and even in a top $B$ background.

351 Following the results attained by PCR experiments, the deleted material in the excision events

352 would remain as a non-replicative circle, as has been reported for other genetic elements that

353 exhibit RecA-independent mobility (Palmieri, Mingoia \& Varaldo, 2013). However, we could

354 not detect the excised form in vivo in none of the two models analyzed. Obviously, this aspect

355 deserves more studies.

356 In sum, we provide new evidence on the occurrence of spontaneous events of excision in E. coli

357 K12 which are not mediated by the known mechanisms of recombination. The rearrangements

358 under study appear to be related to previous descriptions of RecA-independent recombination 
359 events in E. coli K12 (Azpiroz, Bascuas \& Laviña, 2011; Bzymek \& Lovett, 2001; Kingston et

360 al., 2015) and in other bacterial organisms (Santiviago et al., 2010; Palmieri et al., 2012). This

361 type of genetic exchange occurs between regions of homology, including very short ones on

362 which RecA-dependent recombination would not be able to work or would not be efficient.

363 Since its frequency appears to be particularly low, PCR-detection of the recombination products

364 is being routinely used by several authors, while obtaining clones carrying a recombined form

365 has been achieved in very few cases. In this sense, RecA-independent recombination in $\mathrm{Hfr}_{\mathrm{x}} \mathrm{F}^{-}$

366 crosses of extensive chromosomal segments have been reported (Kingston et al., 2015).

367 Interestingly, in a recent article, these authors describe results suggesting that more than one

368 mechanism could contribute to these RecA-independent recombination phenomena (Kingston et

369 al., 2017). In this work we present PCR-based evidence of RecA-independent recombination in

370 two genetic models while in vivo evidence of this phenomenon was attained in only one of them.

371 Finally, it should be kept in mind that if RecA-independent recombination were able to operate

372 on a wide spectrum of repeated sequences, including very short ones, then it would provide

373 mobility to different genetic elements, thus broadening the repertoire of possible rearrangements

374 in the bacterial cell.

375

376 Acknowledgements

377 We are indebted to María Parente for excellent technical assistance.

379 References 
1. Albertini AM, Hofer M, Calos MP, Miller JH. 1982. On the formation of spontaneous deletions: the importance of short sequence homologies in the generation of large deletions. Cell 29: 319-328.

2. Azpiroz MF, Bascuas T, Laviña M. 2011. Microcin H47 system: an Escherichia coli small genomic island with novel features. PloS One 6: e26179.

3. Baba T, Ara T, Hasegawa M, Takai Y, Okumura Y, Baba M, Datsenko KA, Tomita M, Wanner BL, Mori H. 2006. Construction of Escherichia coli K-12 in-frame, single-gene knockout mutants: the Keio collection. Mol Syst Biol 2: 2006.0008.

4. Bi X \& Liu LF. 1994. recA-independent and recA-dependent intramolecular plasmid recombination: differential homology requirement and distance effect. J Mol Biol 235: 414-423.

5. Bzymek M \& Lovett ST. 2001. Instability of repetitive DNA sequences: the role of replication in multiple mechanisms. Proc Natl Acad Sci 98: 8319-8325.

6. Datsenko KA \& Wanner BL. 2000. One-step inactivation of chromosomal genes in Escherichia coli K-12 using PCR products. Proc Natl Acad Sci USA 97: 6640-6645.

7. Hallet B \& Sherratt DJ. 1997. Transposition and site-specific recombination: adapting DNA cut-and-paste mechanisms to a variety of genetic rearrangements. FEMS Microbiol Rev 21: 157-178.

8. Hochhut B, Wilde C, Balling G, Middendorf B, Dobrindt U, Brzuszkiewicz E, Gottschalk G, Carniel E, Hacker J. 2006. Role of pathogenicity island-associated integrases in the 
genome plasticity of uropathogenic Escherichia coli strain 536. Mol Microbiol 61:584-

401 595.

402

403

404

405

406

407

408

409

410

411

412

413

414

415

416

417

418

419

9. Juhas M, van der Meer JR, Gaillard M, Harding RM, Hood DW, Crook DW. 2009.

Genomic islands: tools of bacterial horizontal gene transfer and evolution. FEMS Microbiol Rev 33: 376-393.

10. Kingston AW, Roussel-Rossin C, Dupont C, Raleigh EA. 2015. Novel recA-independent horizontal gene transfer in Escherichia coli K-12. PloS One 10: e0130813.

11. Kingston AW, Ponkratz C, Raleigh EA. 2017. Rpn (YhgA-like) proteins of Escherichia coli K12 and their contribution to RecA-independent horizontal transfer. J. Bacteriol 199: e00787-16.

12. Kolodner R, Hall SD, Luisi-DeLuca C. 1994. Homologous pairing proteins encoded by the Escherichia coli recE and recT genes. Mol Microbiol 11: 23-30.

13. Laviña M, Gaggero C, Moreno F. 1990. Microcin H47, a chromosome-encoded microcin antibiotic of Escherichia coli. J Bacteriol 172: 6585-6588.

14. Lin T, Lee C, Hsieh P, Tsai S, Wang J. 2008. Characterization of integrative and conjugative element ICEKpl-associated genomic heterogeneity in a Klebsiella pneumoniae strain isolated from a primary liver abscess. J Bacteriol 190: 515-526.

15. Lovett ST, Drapkin PT, Sutera VA, Gluckman-Peskind TJ.1993. A sister-strand exchange mechanism for $\operatorname{rec} A$-independent deletion of repeated DNA sequences in Escherichia coli. Genetics 135: 631-642. 
420

421

422

423

424

425

426

427

428

429

430

431

432

433

434

435

436

437

438

439
16. Lovett ST, Gluckman TJ, Simon PJ, Sutera Jr VA, Drapkin PT. 1994. Recombination between repeats in Escherichia coli by a recA-independent, proximity-sensitive mechanism. Mol Gen Genet 245: 294-300.

17. Menard KL \& Grossman AD. 2013. Selective pressures to maintain attachment site specificity of integrative and conjugative elements. PLoS Genet 9: e1003623.

18. Murphy RA \& Boyd EF. 2008. Three pathogenicity islands of Vibrio cholerae can excise from the chromosome and form circular intermediates. $J$ Bacteriol 190: 636-647.

19. Palmieri C, Mingoia M, Massidda O, Giovanetti E, Varaldo PE. 2012. Streptococcus pneumoniae transposon $\operatorname{Tn} 1545 / \operatorname{Tn} 6003$ changes to Tn6002 due to spontaneous excision in circular form of the erm(B)- and aphA3-containing macrolide-aminoglycosidestreptothricin (MAS) element. Antimicrob Agents Chemother 56: 5994-5997.

20. Palmieri C, Mingoia M, Varaldo PE. 2013. Unconventional circularizable bacterial genetic structures carrying antibiotic resistance determinants. Antimicrob Agents Chemother 57: 2440-2441.

21. Poey ME, Azpiroz MF, Laviña M. 2006. Comparative analysis of chromosome-encoded microcins. Antimicrob Agents Chemother 50: 1411-1418.

22. Santiviago CA, Blondel CJ, Quezada CP, Silva CA, Tobar PM, Porwollik S, McClelland M, Andrews-Polymenis HL, Toro CS, Zaldívar M, Contreras I. 2010. Spontaneous excision of the Salmonella enterica serovar Enteritidis-specific defective prophage-like element $\varphi$ SE14. J Bacteriol 192: 2246-2254. 
23. Schofield MA, Agbunag R, Michaels ML, Miller, JH. 1992. Cloning and sequencing of Escherichia coli mutR shows its identity to topB, encoding topoisomerase III. J Bacteriol 174: $5168-5170$.

24. Uematsu N, Eda S, Yamamoto K. 1997. An Escherichia coli topB mutant increases deletion and frameshift mutations in the supF target gene. Mutat Res 383: 223-230.

25. Wang X, Kim Y, Ma Q, Hong SH, Pokusaeva K, Sturino JM, Wood TK. 2010. Cryptic prophages help bacteria cope with adverse environments. Nat Commun 1: DOI: 10.1038/ncomms 1146 .

26. Wang GCY and Wang Y. 1997. Frequency of formation of chimeric molecules as a consequence of PCR coamplification of 16S rRNA genes from mixed bacterial genomes. Applied Environ Microbiol 63: 4645-4650.

27. Whoriskey SK, Schofield MA, Miller JH. 1991. Isolation and characterization of Escherichia coli mutants with altered rates of deletion formation. Genetics 127:21-30. 


\section{Table 1. Conditions of PCR reactions ${ }^{(a)}$}

\begin{tabular}{|c|c|c|c|c|c|}
\hline Model & $\begin{array}{l}\text { Primer } \\
\text { name }^{(b)}\end{array}$ & Primer sequence $5^{\prime} 3^{\prime}$ & $\begin{array}{c}\text { Annealing } \\
\text { temperature }\end{array}$ & $\begin{array}{l}\text { DNA template } \\
\text { (ng) }\end{array}$ & $\begin{array}{l}\text { Product name and } \\
\text { expected size (bp) }\end{array}$ \\
\hline \multirow{4}{*}{$\begin{array}{l}\mathrm{H} 47 \mathrm{Gl} \\
\text { excision from } \\
\text { pEX2000 }\end{array}$} & out1 & CCGTTCATTTTCCTGCTGACCC & & & \\
\hline & out2 & TCTGTTGCCCGTTGATGTTTCCT & $58^{\circ}$ & $200-400$ & OUT $_{H 47}(314-317)$ \\
\hline & in1 & GTTTGTAGGAGCTTTCTTTTTTG & & & \\
\hline & $\ln 2$ & CGCTGATGACTGTTTTTATGTTG & $53^{\circ}$ & $200-400$ & $\mathrm{IN}_{\mathrm{H} 47}(761-764)$ \\
\hline \multirow{4}{*}{$\begin{array}{l}\text { cat } \\
\text { excision from } \\
\text { pUY-FRT }\end{array}$} & $\mathrm{F}$ & GTTGTAAAACGACGGCCAGT & & & \\
\hline & $\mathrm{R}$ & CACAGGAAACAGCTATGACC & $58^{\circ}$ & $200-300$ & OUT $_{\text {FRT }}(381)$ \\
\hline & II-in1 & AAGGCGACAAGGTGCTGATG & & & \\
\hline & II-in2 & GGAACCTCTTACGTGCCGAT & $58^{\circ}$ & $200-300$ & $\mathrm{IN}_{\mathrm{FRT}}(335)$ \\
\hline
\end{tabular}

(a) PCR amplifications were performed using U-Taq DNA polymerase (SBS Genetech) in a total volume of $30 \mu$ l. Reaction mixes contained $1 \mathrm{x}$ buffer, $200 \mu \mathrm{M}$ of each deoxynucleotide triphosphate, $500 \mathrm{nM}$ of each primer, $1.25 \mathrm{U}$ of DNA polymerase and template DNA. Conditions for amplification were: 2 min at $94^{\circ} \mathrm{C}, 30$ cycles of incubation at $94^{\circ} \mathrm{C}$ for $30 \mathrm{sec}$, annealing temperature for 30 sec, $72^{\circ} \mathrm{C}$ for $30 \mathrm{sec}$, and a final extension step at $72^{\circ} \mathrm{C}$ for $2 \mathrm{~min}$.

(b) Primers used to assay the $\mathrm{H} 47 \mathrm{GI}$ model were presented in Azpiroz et al. 2011. F and R are M13 forward and reverse primers. IIin1 and II-in2 were designed in this work. 
A

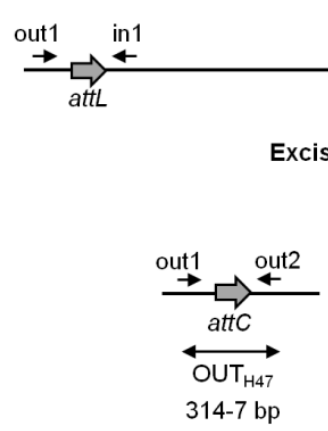

508

509

510

511

512

513

514

515

516

517

518

519

520

B

C

$$
\begin{aligned}
& \text { attl CATTCACATTGTTTATAACTGGCATTACACOGGTGTTGATGCGCACCTTC } \\
& \text { att } R \text { CATATACTCTGAATAAAACCATCATTTCA--GGTGTTGATGCGCGCTTTA }
\end{aligned}
$$

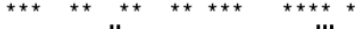

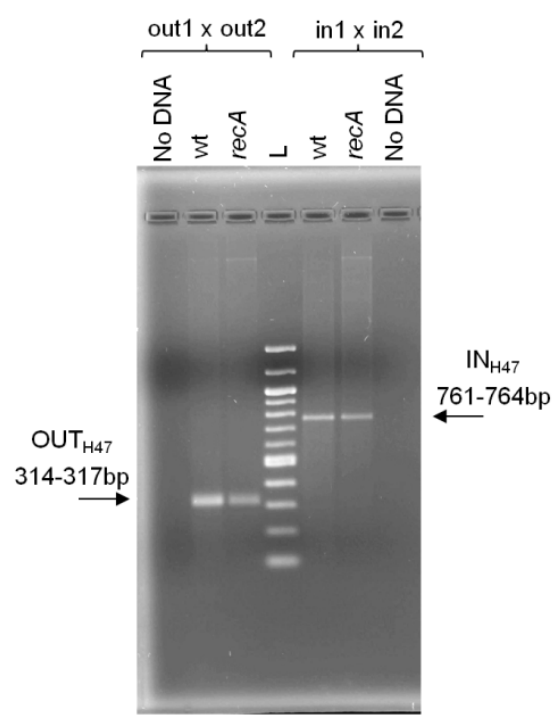


523 Figure 1. Excision of the $\mathbf{H 4 7}$ genomic island. A, PCR approach performed to detect the 524 excision of the H47 GI. Attachment sites are shown with grey thick arrows and primers with 525 small arrows. Products of excision were detected by the appearance of amplicons obtained with 526 the indicated primer pairs: amplicon $\mathrm{OUT}_{\mathrm{H} 47}$, containing the attC site, and amplicon $\mathrm{IN}_{\mathrm{H} 47}$, 527 containing the recombined attachment site attI in the excised H47 GI. B, Alignment of the attL 528 and $a t t R$ sites. Identical nucleotides are indicated with asterisks. The four main regions of 529 homology are boxed and named with roman numbers. C, Detection of the H47 GI excision using 530 as template pEX2000 propagated in E. coli K12 BZB1011 (wt), and in its derivative mutant 531 recA. L: 100 pb DNA Ladder (BioLabs). 
A

543

544

545

546

547

548

549

550

551

552

553 Figure 2. Excision of the cat gene in pUY-FRT and generation of pUY-FRT $\Delta$. A, Plasmid

554 DNA extracted from strains BW25113 recA (pUY-FRT) and BW25113 topB recA (pUY-FRT).

555 Bands corresponding to the original plasmid pUY-FRT $(3,864 \mathrm{bp})$ and to its deletion derivative 
556 pUY-FRT $\Delta(2,932 \mathrm{bp})$ are indicated with arrows. B, Restriction analysis of pUY-FRT and pUY-

557 FRT $\Delta$. L: $1 \mathrm{~kb}$ DNA Ladder (BioLabs).

558

559 